\title{
Registro de espoliação em humano pelo morcego vampiro de asas brancas Diaemus youngi (Jentink, 1893) (Chiroptera: Desmodontinae)
}

\author{
Record of human spoliation by white-winged vampire bat Diaemus youngi (Jentink, 1893)
}

(Chiroptera: Desmodontinae)

\author{
Registro de despojo humano por parte del murciélago vampiro de alas blancas Diaemus youngi \\ (Jentink, 1893) (Chiroptera: Desmodontinae)
}

Recebido: 09/09/2021 | Revisado: 15/09/2021 | Aceito: 16/09/2021 | Publicado: 18/09/2021

\author{
Luiz Augustinho Menezes da Silva \\ ORCID: https://orcid.org/0000-0003-3776-5202 \\ Universidade Federal de Pernambuco, Brasil \\ E-mail: luiz.augustinho@ufpe.br \\ Rosângela Margarida da Silva \\ ORCID: https://orcid.org/0000-0002-6697-0603 \\ Universidade Federal de Pernambuco, Brasil \\ E-mail: rosangela.margarida@ufpe.br \\ Jailson Lúcio dos Santos \\ ORCID: https://orcid.org/0000-0002-3169-4792 \\ Universidade Federal de Pernambuco, Brasil \\ E-mail: jailson.lucio@ufpe.br \\ José Lindemberg Martins Machado \\ ORCID: https://orcid.org/0000-0002-2191-4245 \\ Laboratório Central de Saúde Pública de Pernambuco, Brasil \\ E-mail: debergmachado@hotmail.com \\ Marcos Alexandre Barboza de Santana \\ ORCID: https://orcid.org/0000-0001-5156-6993 \\ Médico Veterinário, Brasil \\ E-mail: marcos.santana_vet@yahoo.com.br \\ Andrea Lopes de Oliveira \\ ORCID: https://orcid.org/0000-0003-3783-6453 \\ Prefeitura Municipal do Ipojuca, Brasil \\ E-mail: andrealopes_vet@ hotmail.com \\ Eduardo José da Silva \\ ORCID: https://orcid.org/0000-0003-3498-7205 \\ Prefeitura Municipal do Ipojuca, Brasil \\ E-mail: eduardosilva0287@yahoo.com \\ Juliana Carla Serafim da Silva \\ ORCID: https://orcid.org/0000-0002-0201-8883 \\ Prefeitura Municipal do Ipojuca, Brasil \\ E-mail: serafim.juliana@gmail.com
}

\begin{abstract}
Resumo
Dentre as mais de 1.400 espécies de morcegos catalogadas no mundo, somente três se alimentam de sangue (Hematofagia). Elas só são encontradas nas Américas (principalmente latina) com ampla distribuição no Brasil. A atividade alimentar de morcegos hematófagos em humanos só era conhecida para Desmodus rotundus, entretanto, em 2016, foi relatada para Diphylla ecaudata. O presente trabalho descreve, pela primeira vez, a utilização de sangue humano em condições de campo por Diaemus youngi. O caso ocorreu na zona rural do município de Ipojuca (08 $23^{\prime} 56^{\prime \prime}$ de latitude sul e 3503'50" de longitude oeste), na Região Metropolitana do Recife, estado de Pernambuco. Um morador de 44 anos relatou que, na madrugada do dia 01 de novembro de 2018, acordou e percebeu um morcego fazendo a sangria em seu braço, e ele matou o animal. Ao amanhecer, procurou um posto de saúde e levou o morcego. O paciente foi encaminhado para as medidas profiláticas adequadas, recebeu soro e vacina, e o morcego foi enviado ao LACEN/PE para diagnose e exame rábico. O exemplar foi diagnosticado com base em suas características morfológicas (manchas brancas nas asas, glândulas bucais, almofadas do polegar, entre outras) como Diaemus youngi (macho; adulto). Depois de feitas a análise da imunofluorescência direta e a prova biológica, testou negativo para o vírus rábico. Este relato demonstra que é preciso aprofundar estudos ecológicos sobre a espécie, principalmente relacionados aos itens alimento e comportamento, bem como sobre os impactos ambientais que possam alterar a seleção de suas presas.
\end{abstract}


Palavras-chave: Atividade alimentar; Dieta; Epidemiologia; Hematófagos; Raiva; Saúde pública.

\begin{abstract}
Among the more than 1400 species of bats cataloged in the world, only three feed on blood (Hematophagy), these only occur in the Americas (mainly Latin) with wide distribution in Brazil. The feeding activity of vampire bats in humans was described only for Desmodus rotundus, however, in 2016 it was reported for Diphylla ecaudata. Thus, this report describes for the first time the use of human blood under fieldwork conditions by Diaemus youngi. The case occurred in the rural area of the municipality of Ipojuca $\left(08^{\circ} 23^{\prime} 56^{\prime \prime S}\right.$ and $\left.35^{\circ} 03^{\prime} 50^{\prime \prime} \mathrm{W}\right)$ in the Metropolitan Region of Recife, state of Pernambuco. A 44-year-old resident reported that at dawn on November 1, 2018, he woke up and noticed a bat bleeding his arm, which killed the animal. At dawn, he looked for a health center taking the bat, the patient was referred for appropriate prophylactic measures, receiving serum and vaccine while the bat was sent to LACEN/PE for diagnosis and rabies examination. The specimen was diagnosed based on its morphological characteristics (white spots on the wings, buccal glands, thumb pads, among others) as Deaemus youngi (male; adult). After analysis of direct immunofluorescence and Biological Proof, tested negative for rabies virus. This report highlights the need to deepen ecological studies on the species, mainly related to food items and behavior, as well as on the environmental impacts that may alter the selection of its prey.
\end{abstract}

Keywords: Eating activity; Diet; Epidemiology; Hematophages; Rabies; Public health.

\begin{abstract}
Resumem
Entre las más de 1400 especies de murciélagos catalogadas en el mundo, solo tres se alimentan de sangre (Hematofagia), estas solo ocurren en las Américas (principalmente latinoamérica) con amplia distribución en Brasil. La actividad de alimentación de los murciélagos vampiros en humanos se describió solo para Desmodus rotundus, sin embargo, en 2016 se informó para Diphylla ecaudata. Así, este informe describe por primera vez el uso de sangre humana en condiciones de campo por Diaemus youngi. El caso ocurrió en la zona rural del municipio de Ipojuca (08²3'56" S y 3503'50" O) en la Región Metropolitana de Recife, estado de Pernambuco. Un residente de 44 años informó que en la madrugada del 1 de noviembre de 2018, se despertó y notó que un murciélago le sangraba el brazo, lo que mató al animal. En la madrugada buscó un centro de salud que tomara el murciélago, el paciente fue referido para las medidas profilácticas oportunas, recibiendo suero y vacuna mientras que el murciélago era enviado a LACEN/PE para diagnóstico y examen de rabia. El espécimen fue diagnosticado por sus características morfológicas (manchas blancas en las alas, glándulas bucales, pulgares, entre otras) como Diaemus. youngi (macho; adulto). Después del análisis de inmunofluorescencia directa y prueba biológica dio negativo para el virus de la rabia. Este informe destaca la necesidad de profundizar los estudios ecológicos sobre la especie, principalmente relacionados con los alimentos y el comportamiento, así como sobre los impactos ambientales que pueden alterar la selección de sus presas.
\end{abstract}

Palabras clave: Actividad alimentaria; Dieta; Epidemiología; Hematófago; Rabia; Salud pública.

\title{
1. Introdução
}

Dentre as espécies de morcegos, destacam-se as hematófagas, devido às suas complexas características morfológicas, fisiológicas e uma singular estrutura social (Aguiar et al., 2006), além de seus hábitos alimentares peculiares restritos ao consumo de sangue de aves e mamíferos (Gardner, 1977). Entretanto, esses animais representam uma pequena parcela da fauna mundial de morcegos, uma vez que, das mais de 1.400 espécies que existem no mundo (Simmons \& Cirranello, 2020), apenas três - Desmodus rotundus (E. Geoffroyi, 1810), Diaemus youngi (Jentink, 1893) e Diphylla ecaudata (Spix, 1823) - têm o sangue como recurso alimentar, todas da subfamília Desmodontinae (Oliveira et al., 2017). Esta subfamília só existe nas Américas e se distribue do Norte do México ao Norte da Argentina (Koopman, 1988; Wilson et al., 2019). Das três espécies, a D. rotundus é a que apresenta a mais ampla distribuição, seguida da D. youngi e da D. ecaudata (Kwon \& Gardner, 2007; Scheffer et al., 2015). De acordo com Uieda (2007), a partir da introdução de animais domésticos no Novo Mundo, as populações de morcegos hematófagos aumentaram e ampliaram suas áreas de ocorrência. Atualmente, a D. rotundus é a mais comum e abundante, ao longo de sua distribuição geográfica, e a mais rara é a D. youngi.

No Brasil, os morcegos hematófagos existem em todos os biomas, porém o D. rotundus é o mais recorrente, com registros em todos os estados e biomas, seguido do D. ecaudata e do D. youngi (Oliveira et al., 2017). Para esta última, existem registros nos estados do Acre, de Alagoas, Amazonas, Amapá, Bahia, Distrito Federal, Goiás, Minas Gerais, Mato Grosso, 
Mato Grosso do Sul, Pará, Paraná, Pernambuco, Rio de Janeiro e São Paulo e Rio Grande do Sul (Oliveira et al., 2017; Pedroso et al., 2018; Hoppe et al., 2019; Witt et al., 2019).

Apesar de o consumo de sangue ser universal para as três espécies, elas podem diferir em relação ao grupo de presas consumidas e nas estratégias comportamentais para acessar essas presas. Sendo assim, a D. rotundus é a espécie mais generalista, pois inclui em sua dieta tanto o sangue de mamíferos quanto o de aves. Já a D. ecaudata e a D. youngi consomem, preferencialmente, sangue de aves (Gardner, 1977; Oliveira et al., 2017). Registros de consumo de sangue de mamíferos para estas duas últimas espécies já foram confirmados em condições de cativeiro (Scheffer et al., 2015). Em relação à ecologia alimentar, a espécie D. youngi é considerada especialista no consumo de aves empoleiradas e de pássaros próximo ao solo (Uieda, 2007; Costa et al., 2008).

Aparentemente, devido aos seus hábitos alimentares, esses animais são perseguidos pela população, sofrem com a discriminação e são, frequentemente, alvos de controle e de manejo inadequados. Vale salientar que a população, em geral, não entende que consumir sangue é apenas um padrão alimentar como outro qualquer e que não deve ser utilizado como referencial para desqualificar sua importância ambiental. Por causa da falta de informações sobre a importância dos morcegos e da má percepção que a população tem sobre esses animais, é comum encontrar agrupamentos de morcegos serem eliminados tanto em seus ambientes naturais quanto em áreas urbanas independentemente dos benefícios que possa proporcionar. A perseguição aos morcegos hematófagos e o manejo/monitoramento inadequado podem acarretar na dispersão de suas populações se estabelecendo em outras áreas e impactos negativos em grupos de morcegos não hematófagos.

No Brasil, os morcegos hematófagos são encontrados, principalmente, em ambientes florestais e/ou nas zonas rurais onde a disponibilidade de presas e de abrigos é maior, o que facilita o estabelecimento de suas populações. Entretanto $D$. rotundus (Dantas-Torres et al., 2005; Ferraz et al., 2007; Nunes et al., 2017), D. ecaudata e D. youngi (Nunes et al., 2017) já foram registradas em áreas urbanas. As duas últimas são associadas a fragmentos florestais urbanos. A presença dessas espécies em áreas antropizadas/urbanizadas pode favorecer o consumo de sangue humano e de animais domésticos (cães e gatos), caso o acesso a esses recursos seja facilitado e outras fontes alimentares não estejam disponíveis (Schneider et al., 2001; Carneiro et al., 2005; Rigo \& Honer, 2005; Lima, 2020). A presença de populações de morcegos hematófagos em áreas urbanas serve como alerta para a vigilância epidemiológica, porque, se estiverem contaminados com o vírus rábico, podem funcionar como veículos de transmissão da doença a humanos (Ferreira et al., 2020).

Os registros de atividade alimentar em humanos por morcegos hematófagos só eram conhecidos para $D$. rotundus (Schneider et al., 2009; Scheffer et al., 2015), até 2016, quando foi registrado o consumo de sangue humano por D. ecaudata no sertão pernambucano (Ito et al., 2016). Vale salientar que os relatos de atividade alimentar de morcegos hematófagos em humanos não são frequentes, e quando ocorrem, geralmente estão associados a imposições ambientais que causaram o declínio de suas presas e/ou facilitaram o acesso aos humanos. Infelizmente, durante os eventos de atividade alimentar de morcegos hematófagos em humanos, a raiva pode ser transmitida (Wada et al., 2004). Nos anos de 2004 e 2005 , os morcegos hematófagos foram os principais transmissores da raiva humana na América Latina, e o Brasil foi o país com o maior número de casos - 64 humanos infectados pelo vírus rábico por morcegos hematófagos (Kotait et al., 2007).

A partir do exposto, este trabalho relata, pela primeira vez, um evento de atividade alimentar em humanos por $D$. youngi, incluindo esse recurso alimentar em sua dieta na natureza.

\section{Metodologia}

\subsection{Descrição da Área}


O caso ocorreu no município de Ipojuca, na mesorregião metropolitana de Recife e na microrregião de Suape, a 49 km da capital (08²3'56" de latitude sul e 3503'50" de longitude oeste), a uma altitude de 10 metros (Figura 12. O município apresenta $527.107 \mathrm{Km}^{2}$ de território e um grau de urbanização de $68 \%$. A maior parte do território compreende a zona rural com, aproximadamente, $515 \mathrm{~km}^{2}$, e o restante ocupado pela área urbana $0,368 \mathrm{~km}^{2}$. Com uma população estimada em 94.709 habitantes, ocupa o $15^{\circ}$ lugar em relação ao número de habitantes no Estado (IBGE, 2019).

Poucos fragmentos florestais de Mata Atlântica se mantêm presentes na área, porquanto grande parte foi suprimida pelo cultivo da cana-de-açúcar. Na zona rural, é comum encontrar pequenos agrupamentos de casas distribuídas de forma espaçada, formando pequenas áreas cultiváveis e de criação de pequenos animais (Figura 1).

Figura 1: Localização do munícipio de Ipojuca no estado de Pernambuco e do local onde ocorreu o registro da atividade alimentar.

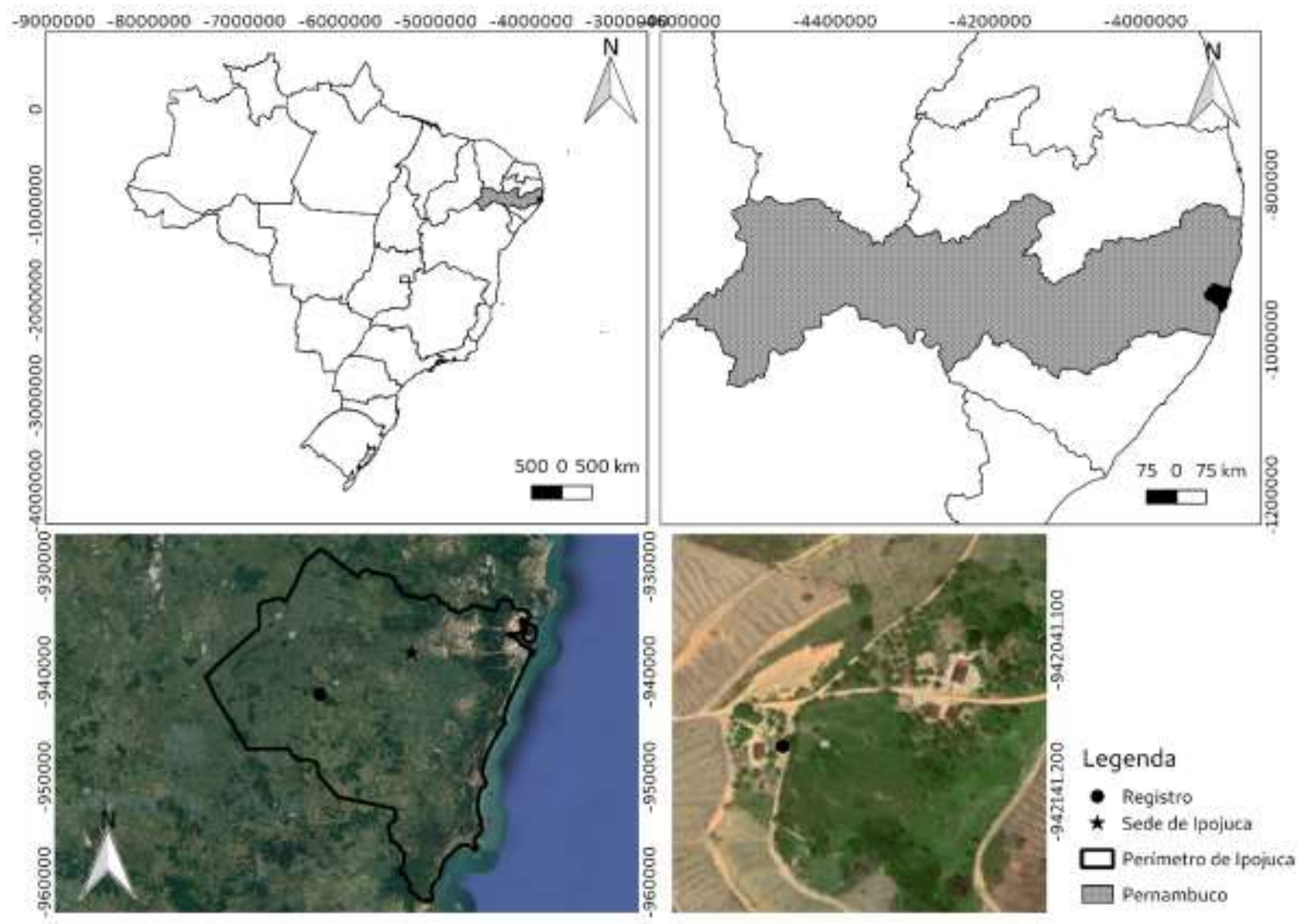

Fonte: Autores.

\subsection{Registro e Diagnose do Caso}

O relato é de natureza qualitativa seguindo as orientações de Turato (2005). Na madrugada de 01/11/2018, um morador de 44 anos da zona rural acordou com um morcego se alimentando em seu braço. Ele matou o animal e, na manhã seguinte, recolheu-o e levou-o para uma unidade básica de saúde. O morcego foi recebido pela Vigilância Ambiental do Município e encaminhado ao Laboratório Central de Saúde Pública/PE (LACEN/PE) para a diagnose e investigação da raiva. A identificação do morcego seguiu as orientações de Diaz et al. (2016) e Oliveira et al. (2017), e o exame para o diagnóstico da presença do vírus rábico seguiu o protocolo estabelecido por Brasil (2008).

\section{Resultados e Discussão}


O registro da agressão ocorreu na zona rural do município de Ipojuca no Engenho Queluz (Figura 1). O munícipe que teve contato com o morcego foi encaminhado para medidas profiláticas, seguindo as orientações de Brasil (2011). Ele recebeu $15 \mathrm{ml}$ de soro heterólogo e uma dose de vacina antirrábica, no mesmo dia da agressão, e três doses de vacina nos dias 04,08 e 15. Foi acompanhado pela Vigilância Ambiental do município para terminar o tratamento. Todo o procedimento ficou registrado na caderneta de vacinação do paciente e no hospital municipal. O animal foi enviado para ser identificado e para ser submetido a teste para saber se havia vírus rábico (Figura $2 \mathrm{~A}$ ). Depois dos testes de imunofluorescência direta e da prova biológica, seguindo os protocolos estabelecidos em Brasil (2008), o morcego foi diagnosticado como negativo para a raiva.

Com base em características morfológicas, como glândulas bucais bem evidentes (Figura 2 B), coloração branca nas extremidades das asas (Figura $2 \mathrm{C}$ e D), polegar com uma única almofada basal e apenas incisivos inferiores externos bilobados, o exemplar foi identificado como D. youngi (Figura 2). O grau de ossificação de suas falanges indicou que era um animal adulto, e a observação de sua genitália, que era um macho não escrotado.

Figura 2: Características diagnósticas encontradas no espécime analisado que confirmam a espécie Diameus youngi. A Espécime adulto, macho não escrotado; B - seta indica as glândulas bucais; C e D detalhes das manchas brancas na asa (dactiliopatagium).

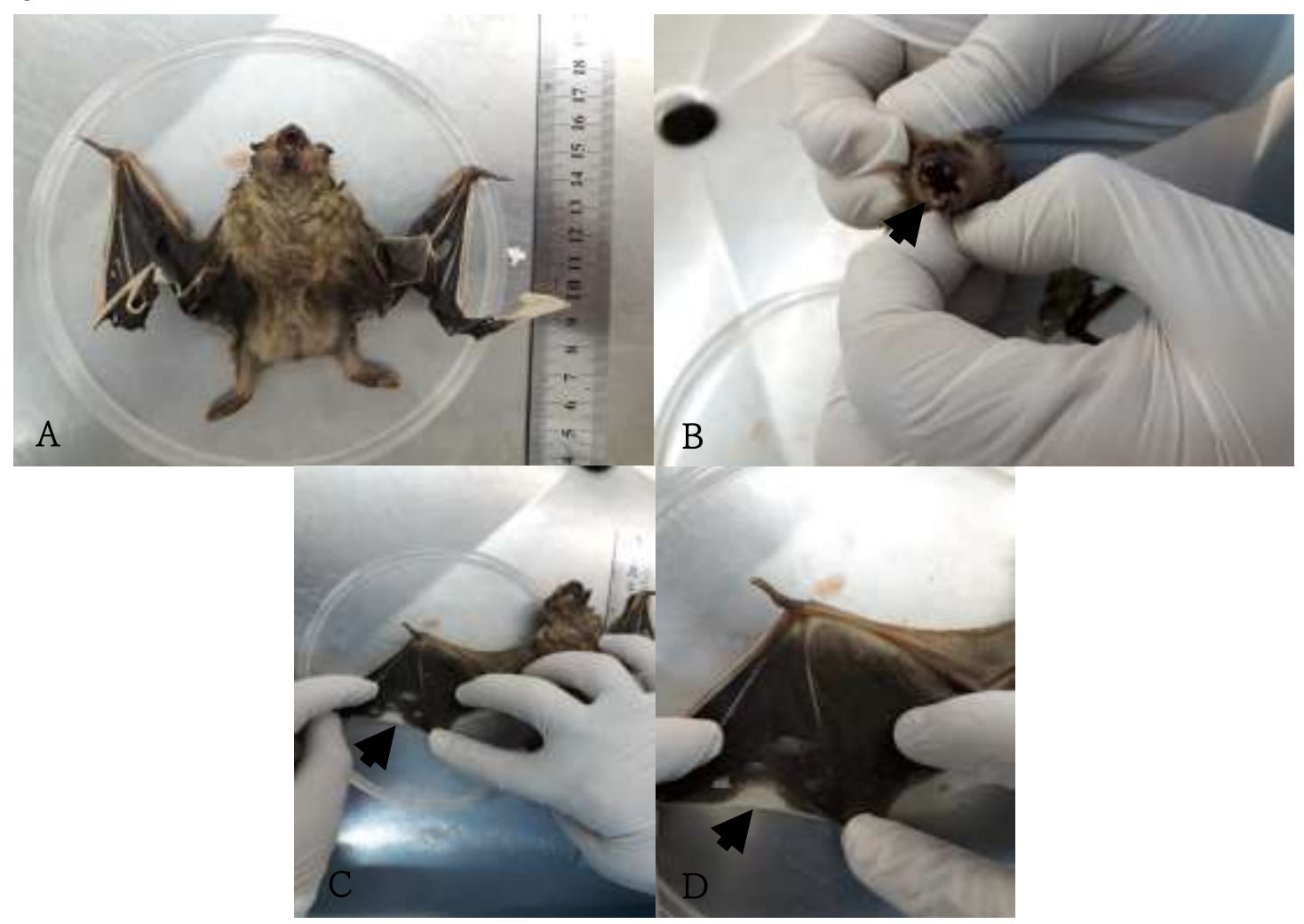

Fonte: Autores.

Apesar de as espécies de morcegos hematófagos compartilharem características diagnósticas em comum (folha nasal em forma de ferradura, polegares desenvolvidos, incisivos em forma de navalha e uropatágio muito reduzido) (Oliveira et al., 2017), de acordo com Diaz et al. (2016) e Oliveira et al. (2017), elas são facilmente separadas, inclusive em condições de campo, observando algumas características mais específicas. Das três espécies, a D. youngi e a D. rotundus são as mais semelhantes, entretanto podem ser distinguidas pela presença de uma única almofada na face ventral no polegar da $D$. youngi, enquanto na $D$. rotundus são três bem desenvolvidas (apresentam esse dígito mais longo). Além dessa característica, a presença de glândulas bucais (tanto nos machos quanto nas fêmeas e são mais evidentes quando o animal está estressado) e as 
manchas brancas nas pontas das asas e no dactiliopatagium (membranas entre os dedos) de D. youngi o separam facilmente de D. rotundus (Scheffer et al., 2015; Diaz et al., 2016; Oliveira et al., 2017).

No Brasil, casos de espoliação em humanos por morcegos hematófagos são registrados em relação à espécie $D$. rotundus, em diferentes localidades do país, alguns em áreas urbanas (Schneider et al., 2009; Fahl et al., 2015). Até recentemente, o uso de sangue humano como fonte de alimento estava restrito a essa espécie, entretanto Ito et al. (2016) referiram o consumo de sangue humano por D. ecaudata em condições naturais, em uma área de caatinga em Pernambuco. Os autores encontraram amostras de sangue humano em fezes acumuladas abaixo do abrigo dos animais, no interior de uma caverna, e nas fezes coletadas diretamente de espécimes capturados que defecaram durante o manuseio. Isso ampliou a lista de presas consumidas e levantou uma discussão sobre a importância dos trabalhos de ecologia alimentar em hematófagos.

Com base na literatura, este trabalho traz o primeiro relato de consumo de sangue humano pela espécie D. young, uma vez que esta espécie consome, exclusivamente, na natureza, sangue de aves (Gardner, 1977; Uieda, 1993; Costa et al., 2008). De acordo com Scheffer et al. (2015), durante a localização das presas, o sentido olfativo é de grande relevância, e os relatos de consumo de sangue de mamíferos por essas espécies ficaram restritos a condições de cativeiro (Scheffer et al., 2015). Em seu trabalho, Scheffer et al. (2015) afirmam que, durante a alimentação, o D. young mantém certa distância da face das aves e se esconde entre as penas e os ramos. Esse comportamento pode estar relacionado ao instinto de defesa contra bicadas. O tempo de alimentação dura, em média, 15 a 30 minutos, e o consumo é em torno de 33ml de sangue (Uieda, 1996).

Ressalte-se, entretanto, que o indicativo desse consumo em condições de cativeiro levanta a possibilidade do uso na natureza. Estudos mais aprofundados sobre a análise do sangue consumido, no conteúdo estomacal ou nas fezes dos morcegos hematófagos, é de fundamental importância para elucidar a amplitude alimentar das espécies em diferentes Biomas, condições de habitat distinto e em áreas antropizadas como visto no trabalho de Ito et al. (2016).

Apesar de a espécie D. youngi ser distribuída amplamente nas Américas, é considerada rara em grande parte dos locais onde é registrada (Koopman, 1988). No Brasil, um número baixo de capturas indica que essa espécie aparenta ser rara no lugar em que existe (Aguiar et al., 2006). Entretanto, a amplitude de sua distribuição geográfica está aumentando (Falcão, 2007; Costa et al., 2008; Feijó et al., 2010; Pedroso et al., 2018; Hoppe et al., 2019; Witt et al., 2019). De acordo com os dados apresentados por Uieda (2007), a D. youngi aparenta ser a espécie de morcego hematófago mais rara devido às suas especializações de hábito alimentar com o consumo de sangue de aves e a utilização de ocos de árvores como abrigo diurno. A exigência desse tipo de abrigo pode limitar a distribuição da espécie associando-a a áreas mais preservadas e que apresentem árvores de grande porte. A condição rara levantada pelos trabalhos para a espécie, atrelada a outras imposições locais sofridas por ela possibilitou que a D. youngi fosse colocada em algumas listas de espécies ameaçadas de extinção (Bergallo et al., 2000; Margarido \& Braga, 2004). No Brasil, essa espécie se distribui ao longo de 13 estados (IUCN, 2021) e é pouco comum na Região Nordeste (Guerra, 2007). Em Pernambuco, a D. rotundus e a D. ecaudata são mais distribuídas, enquanto a D. youngi é registrada em poucas localidades (Guerra, 2007). Em Pernambuco, a espécie D. youngi foi capturada poucas vezes, unicamente em áreas de Mata Atlântica, com uma distribuição restrita aos municípios de São Lourenço da Mata (Mares et al., 1981), Rio Formoso (Guerra, 2007), Jaqueira (Silva et al., 2010) e Ipojuca (Sotero-Caio et al., 2011).

A paisagem em que o evento ocorreu era uma área rural bem antropizada, formada de diferentes sítios, casas distantes e mais isoladas e poucos fragmentos de mata (Figura 3), o que evidencia que essa espécie pode utilizar áreas antropizadas, como já relatado para D. rotudus e D ecaudata (Scheffer et al., 2015, Dantas-Torres et al., 2005, Nunes et al., 2017). Boa parte da vegetação natural (Mata Atlântica) do local onde o registro ocorreu foi suprimida e só existem pequenos fragmentos florestais distribuídos de forma muito esparsa. Na área, predomina o cultivo de cana de açúcar, e nos arredores do sítio, havia animais domésticos. Os impactos ambientais podem favorecer o deslocamento de animais silvestres e a propagação de zoonoses, deixando os seres humanos mais vulneráveis a enfermidades (Tavares et al., 2020). 
Figura 3: Vista geral da paisagem observem pequenos fragmentos florestais de floresta atlântica (capoeiras) próximos a residência.

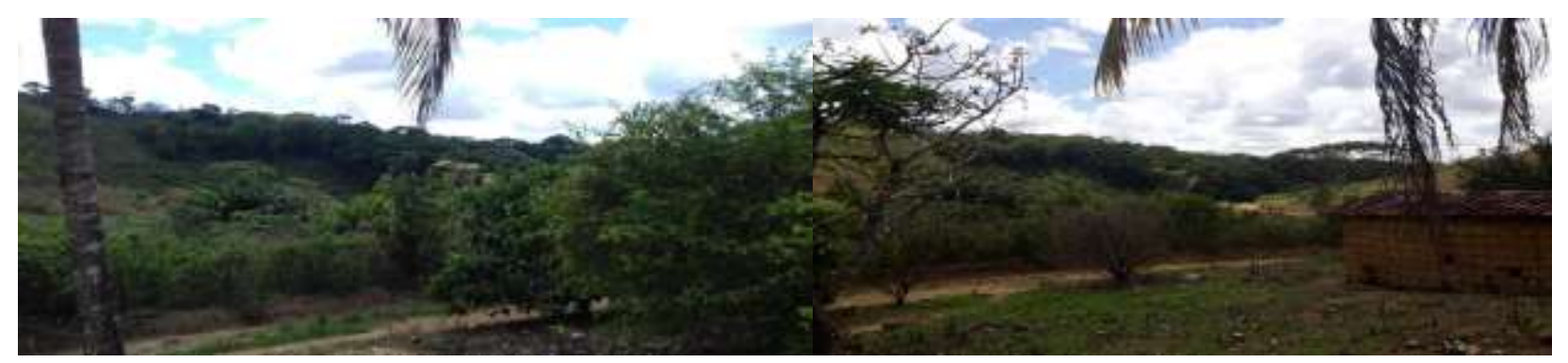

Fonte: Autores.

A residência apresentava um telhado de madeira (caibros e ripas) coberto de telhas tipo cerâmica, em estilo colonial, construída de alvenaria, e cujas paredes tinham o reboco exposto. Havia um espaço (frestas) entre as paredes e o telhado, que, provavelmente, facilitou o adentramento do morcego. Os cômodos eram separados por paredes e cortinas que não chegavam até o teto, e as telhas e os caibros não tinham um forro interno. Aproximadamente 2,5 de altura entre a parte mais alta do teto da casa e o chão (Figura 4).

Figura 4: Local onde ocorreu o registro de atividade alimentar em humanos pelo morcego Diaemus young. A - Parte de trás da residência; B e C - visão lateral; D - Parte da frente da residência; E - Visão interna.

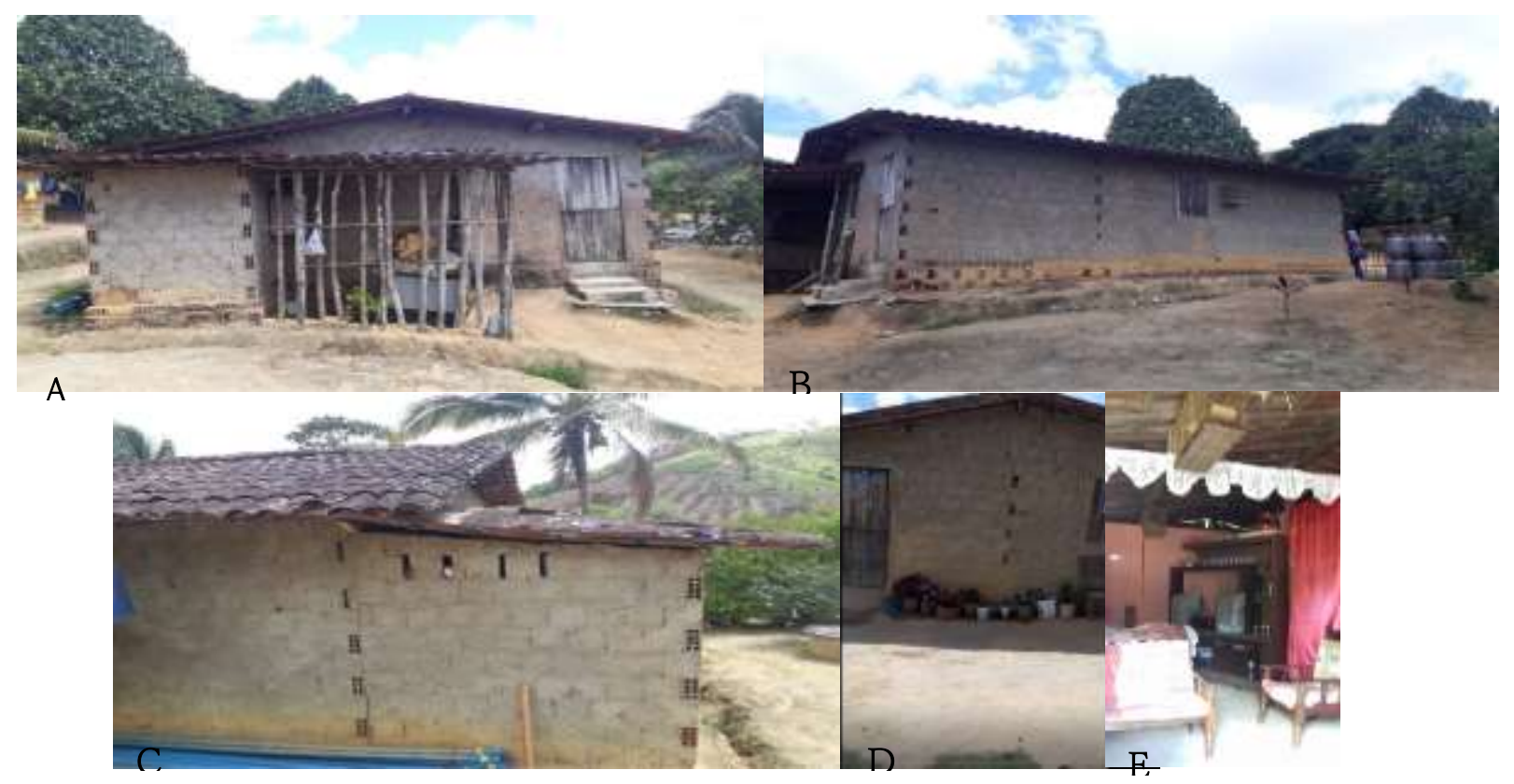

Fonte: Autores.

Apesar de as três espécies de morcegos hematófagos já apresentarem diagnóstico positivo de raiva no Brasil (Sodré, et al., 2010), dentre elas, apenas a D. rotundus é mais importante no ciclo da doença, tanto na raiva humana quanto na dos herbívoros. Isso causa grandes prejuízos econômicos na pecuária (Queiroz, et al., 2009). Sendo assim, devem-se tomar medidas adequadas de manejo ecológico e de controle ao encontrar esses animas em coletas e verificar o indicado para cada 
uma das espécies de hematófagos (IBAMA, 2009; Mialhe \& Moschini, 2016) sem impactar em suas populações.

\section{Considerações Finais}

Relacionando o registro feito neste trabalho e o de Ito et al. (2016) sobre a espécie D. ecaudata também sobre o consumo de sangue de humanos na natureza, podemos afirmar que os hematófagos podem ser mais flexíveis em relação ao tipo de presa consumido, principalmente quando há imposições ambientais. Entretanto, não se devem inserir essas espécies em planos de controle de morcegos hematófagos no monitoramento da raiva, mas desenvolver mais estudos sobre sua ecologia alimentar em ambientes naturais para saber quais são os fatores que levam essas espécies de hematófagos a explorarem novas presas como recurso alimentar. Para melhor conhecer a ecologia alimentar dos morcegos hematófagos é de fundamental importância a realização de trabalhos sobre os tipos de presas consumidas a partir das observações de campo e em coletas nos abrigos.

\section{Agradecimentos}

Às equipes do LACEN/PE - Setor de diagnóstico da raiva e da Vigilância em Saúde do Município de Ipojuca pelo apoio logístico e no estudo do caso.

\section{Referências}

Aguiar, L. M. S., Camargo, W. R. \& Portella, A. S. (2006). Occurrence of white-winged vampire bat, Diaemus youngi (Mammalia, Chiroptera), in the Cerrado of Distrito Federal, Brazil. Revista Brasileira de Zoologia, 23(3), 893-896.

Bergallo, H. G., Geise, L., Bonvicino, C. R., Cerqueira, R., D’Andrea, P. S., Esberard, C. E., Fernandez, F. A. S., Grelle, C. E. V., Siciliano, S. \& Vaz, S. M. (2000). Mamíferos. In H. G. Bergallo, C. F. D. Rocha, M. V. Sluys, L. Geise, M. A. A. (Ed.), A fauna ameaçada de extinção do estado do Rio de Janeiro. (pp. 125-136) Editora da UERJ.

Brasil. (2008). Manual de Diagnóstico Laboratorial da Raiva. Editora do Ministério da Saúde.

Brasil (2011). Normas técnicas de profilaxia da raiva humana. Editora do Ministério da Saúde.

Carneiro, R. L., Martins, M. S., Brasil, T. K., Silva, L. L. A., Santos, E. F., \& Souza, L. E. B. (2005). Análise das agressões em humanos por morcegos hematófagos ocorridas em três municípios baianos no período de 1986 a 1995. Revista Brasileira de Saúde e Produção Animal, 6(1), $1-7$.

Costa, L. M., Oliveira, D. M. D., Fernandes, A. F. P. D., \& Esberard, C. E. L. (2008). Ocorrência de Diaemus youngi (Jentink 1893), Chiroptera, no estado do Rio de Janeiro. Biota Neotropical, 1 (8), 217-220. https://doi.org/10.1590/S1676-06032008000100023

Dantas-Torres, F., Valença, C., \& Andrade Filho, G. V. (2005). Primeiro registro de Desmodus rotundus na área urbana da cidade de Olinda, Pernambuco, Nordeste do Brasil: relato de caso. Revista do Instituto de Medicina Tropical de São Paulo, 47 (2), 107-108. https://www.revistas.usp.br/rimtsp/article/view/30896

Díaz, M. M., Solari, S., Aguirre, L. F., Aguiar, L., \& Barquez, R. M. (2016). Clave de Identificación de los Murciélagos de Sudamérica. Tucumán, Argentina: Publicación Especial n 2.

Fahl, W. O., Garcia, A. L. E., Achkar, S. M., Mori, E., Asano, K. M., lamamoto, K., \& Scheffer, K. C. (2015). Rabia transmitida por murciélagos en Brasil. Acta Biológica Colombiana, 20 (3): 21-35. https://doi.org/10.15446/abc.v20n3.45481

Falcão, F. C. (2007). Mammalia, Chiroptera, Phyllostomidae, Diaemus youngi: First record for the state of Bahia, northeastern Brazil. Check List, 3(4), 330332.

Feijó, A., Araújo, P., Fracasso, M. P. A., \& Santos, K. R. P. (2010). New records of three bat species for the Caatinga of the state of Paraíba, northeastern Brazil. Chiroptera Neotropical, 16(2), 723-727.

Ferraz, C., Achkar, S.M., \& Kotait, I. (2007). Primeiro relato de raiva em morcego hematófago (Desmodus rotundus) em área urbana. Revista do Instituto de Medicina Tropical de São Paulo, 49 (6), 389-390. https://www.revistas.usp.br/rimtsp/article/view/31132

Ferreira, G. S., Oliveira, R. A., Galvão, L. B., Paula, E. M. N., Melo, P. C., Romani, A. F., Ramos, D. G. S., Amaral, A. V. C., Cruz, C. A., Sousa, D. B., \& Bartoli, R. B. M. (2020). Antirrabic coverage rate in dogs and cats in the years of 2010 to 2018 in municipal campaings of Jataí - Goiás, Brazil. Research, Society and Development, 9 (7), 10.33448/rsd-v9i7.4223

Gardner, A. L. (1977). Feeding habits. In Baker, R.J., Jones, J. K. \& Carter, D. C. (Ed.), Biology of bats of the New World Family Phyllostomatidae (pp. 293350). Texas: Tech Uni. 
Guerra, D. Q. (2007). Chiroptera de Pernambuco: distribuição e aspectos biológicos (Dissertação de mestrado). Universidade Federal de Pernambuco - UFPE Recife, PE, Brasil. https://attena.ufpe.br/bitstream/123456789/746/1/arquivo1778_1.pdf

Hoppe, J. P. M., Marchezi, G., \& Dietchfiel, A. D. (2019). First record of the white-winged vampire bat, Diaemus youngii (Jentink, 1893) (Chiroptera, Phyllostomidae) for the state of Goiás, Brazil, with a revised distribution map. Check List the journal of biodiversity data, 15(1) 55-64. https://doi.org/10.15560/15.1.55

Instrução Normativa IBAMA $n^{o}$ 13, de 14 de maio de 2009. Dispõe de sobre alterar a Instrução Normativa IBAMA ${ }^{\circ}$ 171/2008. https://www.icmbio.gov.br/cepsul/images/stories/legislacao/Instrucao_normativa/2009/in_ibama_13_2009_defesotainha_se_s_alt_in_ibama_171_2008.pdf

Instituto Brasileiro Geografia e Estatística. (2019). Cidades e Estados: Ipojuca. ibge.gov.br/cidades-e-estados/pe/ipojuca.html

Ito, F., Bernard, E., \& Torres, R. A. (2016). What is for Dinner? Fist Reporto f Human Blood in the Diet of the Hairy-Legged Vampire Bat Diphylla ecaudata. Acta Chiropterologica, 18 (2) 509-515. https://doi.org/10.3161/15081109ACC2016.18.2.017

International Union for Conservation of Nature's. (2021). The IUCN Red List of Threatened Species. https://www.iucnredlist.org

Koopman, K. F. (1988). Systematics and distribution. In A. M. Greenhall, \& Schmidt, U. (ed.). Natural History of Vampire Bats. (pp. 7-17), CRC Press.

Kotait, I., Carrieri, M. L., Carnieli Júnior, P., Castilho, J. G., Oliveira, R. D. N., Macedo, C. I., \& Achkar, S. M. (2007). Reservatórios silvestres do vírus da raiva: um desafio para a saúde pública. Boletim Epidemiológico Paulista, 4(40), 02-08.

Kwon, M., \& Gardner., A. L. (2007). Subfamily Desmodontinae. In A. L. Gardner (Ed.), Mammals of South America: Marsupials, Xenarthrans, Shrews, and Bats. (2a ed.), 521-523. Chicago: The University of Chicago Press.

Lima, A. P. L. (2020). Agressão por morcegos em humanos em uma área de conservação na Amazônia Oriental (Dissertação de mestrado). Universidade Federal do Pará - UFPA, Castanhal, PA, Brasil. http://repositorio.ufpa.br/jspui/handle/2011/13157.

Mares, M. A., Willig, M. R., Streilein, K. E., \& Lacher, T. (1981). The mammals of northeastern Brazil: a preliminary assessment. Research Gate. 50(4), 81137.

Margarido, T. C. C., \& Braga, F. C. (2004). Mamíferos. In S. B. Mikich, \& R. S. Bérnils (Ed.), Livro vermelho da fauna ameaçada no estado do Paraná (pp. 127-142). Curitiba: Instituto Ambiental do Paraná.

Mialhe, P. J., \& Moschini, L. E. (2016). Controle populacional do morcego hematófago Desmodus rotundus e redução de ataques a herbívoros domésticos no município de São Pedro, São Paulo, Brasil. Journal of Social, Technological and Environmental Science, 5(3): 238- 250. http://dx.doi.org/10.21664/22388869.2016v5i3.p238-251

Nunes, H., Rocha, F. L., \& Cordeiro-Estrela, P. (2017). Bats in urban areas of Brazil: roosts, food resources and parasites in disturbed environments. Urban Ecosystems, 20 (4), 953-969. 10.1007/s11252-016-0632-3

Oliveira, G. R. G. S. Porto \& Lima, I. P. (2017). Subfamília Desmodontinae. In N. R. Reis, A. L. Peracchi, C. B. Batista, \& I. P. Lima, (ed.), História Natural dos Morcegos Brasileiros: Chave de Identificação de Espécies. Rio de Janeiro: Technical Books Editora.

Pedroso, M. A., Rocha, P. A., Brandão, M. A., Garbino, G. S. T., Moraes, C. O. \& Aires, C. C. (2018). Filling gaps in the distribution of the whitewinged vampire bat, Diaemus youngii (Phyllostomidae, Desmodontinae): New records for Southern Amazonia. Acta Amazonica, 48 (2) $154-157$. https://doi.org/10.1590/1809-4392201704291

Queiroz, L. H., Carvalho, C., Buso, D. S., Ferrari, C. I. L., \& Pedro, W. A. (2009). Perfil epidemiológico da raiva na região noroeste do estado de São Paulo no período de 1993 a 2007. Revista da Sociedade Brasileira de Medicina Tropical, 42 (1), 9-14. https://doi.org/10.1590/S0037-86822009000100003

Rigo, L., \& Honer, M. R. (2005). Análise da profilaxia da raiva humana em Campo Grande, Mato Grosso do Sul, Brasil, em 2002. Rio de Janeiro: Cadernos de Saúde Pública, 21 (6): 1939-1945. https://doi.org/10.1590/S0102-311X2005000600044

Scheffer, K. C., Barros, R. F., Iamamoto, K., Mori, E., Asano, K. M., Achkar, S. M., \& Fahl, W. O. (2015). Diphylla ecaudata y Diaemus youngi, Biología y comportamiento. Acta Zoológica Mexicana, 31(3): 436-445. Retrieved from http://www.scielo.org.mx/pdf/azm/v31n3/v31n3a9.pdf

Schneider, M. C., Romijn, P. C., Uieda, W., Tamayo, H., Silva, D. F., Belotto, A., \& Leanes, L. F. (2009). Rabies transmitted by vampire bats to humans: an emerging zoonotic disease in Latin America? Revista Panamericana de Salud Pública, 25 (3), 260-269. https://www.scielosp.org/article/rpsp/2009.v25n3/260269/en/

Schneider, M. C., Aron, J., Santos-Burgoa, C., Uieda, W., \& Ruiz-Velazco, S. (2001). Common vampire bat attacks on humans in a village of the Amazon region of Brazil. Cadernos de Saúde Pública, 17 (6): 1531-1536. https://doi.org/10.1590/S0102-311X2001000600025

Silva, L. A. M., Souza, A. Q. S., Lima, A. S., Araújo, C. S. F., Silva, C. V. M., Silva, L. E. C., \& Silva, R. M. (2010). Morcegos da Reserva Particular do Patrimônio Natural Frei Caneca, nordeste do Brasil. Chiroptero Neotropical 16 (1) 86-89.

Simmons, N. B., \& Cirranello, A. L. (2020). Bat Species of the World: A taxonomic and geographic database. American Museum of Natural History, 7 (10). Retrieved from https://www.batnames.org/

Sodré, M. M., Gama, A. R., \& Almeida, M. F. (2010). Updated list of bat species positive for rabies in Brazil. Revista Instituto Medicina Tropical, 52(2), 7581. https://doi.org/10.1590/S0036-46652010000200003

Sotero-Caio, C. G., Pieczarka, J. C., Nagamachi, C. Y., Gomes, A. J. B., Lira, T. C., O’Brien, P. C. M., \& Santos, N. (2011). Chromosomal homologies among vampire bats revealed by chromosome painting (Phyllostomidae, Chiroptera). Cytogenetic and Genome Research, 132(3), 156-164. https://doi.org/10.1159/000321574 
Tavares, M. J. L., Fernandes, C. S., Maranhão, P. F. A., Rocha, M. B., Costa, P. M. M. (2020). Ações antrópicas e o surgimento de pandemias: qual o papel da engenharia ambiental nesse contexto? Research, Society and Development, 9(8), 1-17. https://doi.org/10.33448/rsd-v9i8.6727

Turato, E. R. (2005). Métodos qualitativos e quantitativos na área da saúde: definições, diferenças e seus objetivos de pesquisa. Revista Saúde Pública, 39(3), 507-514. https://www.scielo.br/j/rsp/a/qtCBFFfZTRQVsCJtWhc7qnd/?format=pdf\&lang=pt

Uieda, W. (1993). Comportamento alimentar do morcego hematófago Diaemus youngi, em aves domésticas. Revista Brasileira de Biologia, 53(4), 529-538.

Uieda, W. (1996). Biologia e dinâmica populacional de morcegos hematófagos. Anais do II Curso de Atualização em raiva dos herbívoros, 63-87. https://dspace.icmbio.gov.br/jspui/handle/cecav/731

Uieda, W. (2007). História natural dos morcegos hematófagos no Brasil. In S. M. Pacheco, R. V. Marques, C. E. L. Esbérard, (Ed.), Morcegos no Brasil: biologia, sistemática, ecologia e conservação (pp. 179-198). Porto Alegre: Armazém Digital.

Wada, M. Y., Begot, A. L., Noronha, S. L. B., Almeida, I. F. D., Lima, R. J. D. S., Santos, L. B. C. D., \& Hatch, D. L. (2004). Surto de raiva humana transmitida por morcegos no Município de Portel-Pará, março/abril de 2004. Boletim Eletrônico Epidemiológico, 4 (6) 1-5. https://antigo.saude.gov.br/images/pdf/2014/julho/16/Ano04-n06-surto-raiva-hum-morcegos-pa-completo.pdf

Wilson, D. E., \& Mittermeier, R. A. (2019). Handbook of the Mammals of the World- Vol. 9, Bats. Lynx Editions: Journal of Vertebrate Biology

Witt, A.A., Mendes, F. C., Grillo, H. C. Z., Willson, H., \& Rossato, N. A. (2019). Primeiro registro de Diaemus youngi (Jentink, 1893) no estado do Rio Grande do Sul, Brasil. Notas sobre Mamíferos Sudamericanos, 1-7. 10.31687/saremNMS.19.0.15 